\title{
Viscosity Projection Algorithms for Pseudocontractive Mappings in Hilbert Spaces
}

\author{
Xiujuan Pan, ${ }^{1,2}$ Shin Min Kang, and Young Chel Kwun ${ }^{4}$ \\ ${ }^{1}$ School of Science, Tianjin Polytechnic University, Tianjin 300387, China \\ ${ }^{2}$ College of Management and Economics, Tianjin University, Tianjin 300072, China \\ ${ }^{3}$ Department of Mathematics and the RINS, Gyeongsang National University, Jinju 660-701, Republic of Korea \\ ${ }^{4}$ Department of Mathematics, Dong-A University, Busan 614-714, Republic of Korea
}

Correspondence should be addressed to Young Chel Kwun; yckwun@dau.ac.kr

Received 6 December 2013; Accepted 28 December 2013; Published 9 February 2014

Academic Editor: Chong Li

Copyright (C) 2014 Xiujuan Pan et al. This is an open access article distributed under the Creative Commons Attribution License, which permits unrestricted use, distribution, and reproduction in any medium, provided the original work is properly cited.

An explicit projection algorithm with viscosity technique is constructed for finding the fixed points of the pseudocontractive mapping in Hilbert spaces. Strong convergence theorem is demonstrated. Consequently, as an application, we can approximate to the minimum-norm fixed point of the pseudocontractive mapping.

\section{Introduction}

Let $\mathbb{Q}$ be a real Hilbert space with inner product $\langle\cdot, \cdot\rangle$ and norm $\|\cdot\|$, respectively. Let $\mathbb{C}$ be a nonempty closed convex subset of $\mathbb{\text { MI}}$.

Recall that a mapping $\mathbb{\mathbb { I }}: \mathbb{C} \rightarrow \mathbb{C}$ is said to be

(i) $L$-Lipschitz $\Leftrightarrow$ there exists a constant $L>0$ such that

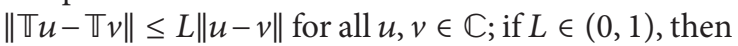
$\mathbb{T}$ is said to be contractive; if $L=1$, then $\mathbb{T}$ is said to be nonexpansive;

(ii) pseudocontractive

$$
\begin{aligned}
& \Leftrightarrow\langle\mathbb{T} u-\mathbb{T} v, u-v\rangle \leq\|u-v\|^{2} \\
& \left.\Leftrightarrow\|\mathbb{T} u-\mathbb{T} v\|^{2} \leq\|u-v\|^{2}+\|(I-\mathbb{T}) u-(I-\mathbb{T}) v\right) \|^{2} ; \\
& \Leftrightarrow\langle u-v,(I-\mathbb{T}) u-(I-\mathbb{T}) v\rangle \geq 0,
\end{aligned}
$$

for all $u, v \in \mathbb{C}$

Interest in pseudocontractive mappings stems mainly from their firm connection with the class of nonlinear accretive operators. It is a classical result, see Deimling [1], that if $\mathbb{T}$ is an accretive operator, then the solutions of the equations $\mathbb{x}=0$ correspond to the equilibrium points of some evolution systems. This explains the importance, from this point of view, of the improvement brought by the
Ishikawa iteration which was introduced by Ishikawa [2] in 1974. The original result of Ishikawa is stated in the following.

Theorem 1. Let $\mathbb{C}$ be a convex compact subset of a Hilbert space $\mathbb{U}$ and let $\mathbb{T}: \mathbb{C} \rightarrow \mathbb{C}$ be an L-Lipschitzian pseudocontractive mapping with $\operatorname{Fix}(\mathbb{T}) \neq \emptyset$. For any $x_{0} \in \mathbb{C}$, define the sequence $\left\{x_{n}\right\}$ iteratively by

$$
\begin{aligned}
& y_{n}=\left(1-\eta_{n}\right) x_{n}+\eta_{n} \rrbracket x_{n}, \\
& x_{n+1}=\left(1-\xi_{n}\right) x_{n}+\xi_{n} \rrbracket y_{n},
\end{aligned}
$$

for all $n \in \mathbb{N}$, where $\left\{\xi_{n}\right\} \subset[0,1]$ and $\left\{\eta_{n}\right\} \subset[0,1]$ satisfy the conditions: $\lim _{n \rightarrow \infty} \eta_{n}=0$ and $\sum_{n=1}^{\infty} \xi_{n} \eta_{n}=\infty$. Then the sequence $\left\{x_{n}\right\}$ generated by (1) converges strongly to a fixed point of $\mathbb{T}$.

The iteration (1) is now referred to as the Ishikawa iterative sequence. However, we note that $C$ is compact subset. Now, we know that strong convergence has not been achieved without compactness assumption on the involved operation or the underlying spaces. A counter example can be found in Chidume and Mutangadura [3].

In order to obtain a strong convergence result for pseudocontractive mappings without the compactness assumption, in [4], Zhou coupled the Ishikawa algorithm with the 
hybrid technique and presented the following algorithm for Lipschitz pseudocontractive mappings:

$$
\begin{aligned}
& y_{n}=\left(1-\xi_{n}\right) x_{n}+\xi_{n} \rrbracket x_{n},
\end{aligned}
$$

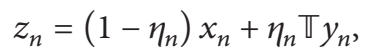

$$
\begin{aligned}
& \mathbb{C}_{n}=\left\{z \in \mathbb{C}:\left\|z_{n}-z\right\|^{2} \leq\left\|x_{n}-z\right\|^{2}\right. \\
& \left.-\xi_{n} \eta_{n}\left(1-2 \xi_{n}-\xi_{n}^{2} L^{2}\right)\left\|x_{n}-\mathbb{\top} x_{n}\right\|^{2}\right\}, \\
& \mathbb{Q}_{n}=\left\{z \in \mathbb{C}:\left\langle x_{n}-z, x_{0}-x_{n}\right\rangle \geq 0\right\}, \\
& x_{n+1}=\operatorname{proj}_{\mathbb{C}_{n} \cap \mathbb{Q}_{n}} x_{0}, \quad n \in \mathbb{N} .
\end{aligned}
$$

Zhou proved that the sequence $\left\{x_{n}\right\}$ generated by (2) converges strongly to the fixed point of $\mathbb{T}$. Further, in [5], Yao et al. introduced the hybrid Mann algorithm as follows.

Let $\mathbb{C}$ be a nonempty closed convex subset of a real Hilbert space $\mathbb{H}$. Let $\left\{\xi_{n}\right\}$ be a sequence in $(0,1)$. Let $x_{0} \in \mathbb{H}$. For $\mathbb{C}_{1}=$ $\mathbb{C}$ and $x_{1}=\operatorname{proj}_{\mathbb{C}_{1}} x_{0}$, define a sequence $\left\{x_{n}\right\}$ of $\mathbb{C}$ as follows:

$$
\begin{aligned}
& y_{n}=\left(1-\xi_{n}\right) x_{n}+\xi_{n} \rrbracket x_{n}, \\
& \mathbb{C}_{n+1}=\left\{z \in \mathbb{C}_{n}:\left\|\xi_{n}(I-\mathbb{T}) y_{n}\right\|^{2}\right. \\
& \left.\leq 2 \xi_{n}\left\langle x_{n}-z,(I-\mathbb{T}) y_{n}\right\rangle\right\}, \\
& x_{n+1}=\operatorname{proj}_{\mathbb{C}_{n+1}} x_{0}, \quad n \in \mathbb{N} .
\end{aligned}
$$

Note that, in iterations (2) and (3), we need to compute the half-spaces $\mathbb{C}_{n}$ (and/or $\left.\mathbb{Q}_{n}\right)$. Very recently, Zegeye et al. [6] further studied the convergence analysis of the Ishikawa iteration (1). They proved ingeniously the strong convergence of the Ishikawa iteration (1). However, we have to assume that the interior of $\operatorname{Fix}(\mathbb{T})$ is nonempty. This appears very restrictive since even in $\mathbb{R}$ with the usual norm, Lipschitz pseudocontractive maps with finite number of fixed points do not enjoy this condition that $\operatorname{int} \operatorname{Fix}(\mathbb{T}) \neq \emptyset$. For some related works, please refer to [7-19].

On the other hand, we notice that it is quite often to seek a particular solution of a given nonlinear problem, in particular, the minimum-norm solution. For instance, given a closed convex subset $\mathbb{C}$ of a Hilbert space $\mathbb{H}_{1}$ and a bounded linear operator $\mathbb{B}: \mathbb{H}_{1} \rightarrow \mathbb{H}_{2}$, where $\mathbb{H}_{2}$ is another Hilbert space. The $\mathbb{C}$-constrained pseudoinverse of $\mathbb{B}, \mathbb{B}_{\mathbb{C}}^{\dagger}$, is then defined as the minimum-norm solution of the constrained minimization problem

$$
\mathbb{B}_{\mathbb{C}}^{\dagger}(b):=\arg \min _{x \in \mathbb{C}}\|\mathbb{B} x-b\|
$$

which is equivalent to the fixed point problem

$$
u=\operatorname{proj}_{\mathbb{C}}\left(u-\mu \mathbb{B}^{*}(\mathbb{B} u-b)\right),
$$

where $\mathbb{B}^{*}$ is the adjoint of $\mathbb{B}, \mu>0$ is a constant, and $b \in \mathbb{H}_{2}$ is such that $\operatorname{proj}_{\overline{\mathbb{B}(\mathbb{C})}}(b) \in \mathbb{B}(\mathbb{C})$.

It is, therefore, an interesting problem to invent iterative algorithms that can generate sequences which converge strongly to the minimum-norm solution of a given fixed point problem. The purpose of this paper is to solve such a problem for pseudocontractions. More precisely, we will introduce an explicit projection algorithm with viscosity technique for finding the fixed points of a Lipschitzian pseudocontractive mapping. Strong convergence theorem is demonstrated. Consequently, as an application, we can find the minimum-norm fixed point of the pseudocontractive mapping.

\section{Preliminaries}

Recall that the metric projection proj $\mathfrak{C}_{\mathbb{C}}: \mathbb{U} \rightarrow \mathbb{C}$ satisfies $\| u-$ $\operatorname{proj}_{\mathbb{C}} u \|=\inf \{\|u-v\|: v \in \mathbb{C}\}$. The metric projection $\operatorname{proj}_{\mathbb{C}}$ is a typical firmly nonexpansive mapping. The characteristic inequality of the projection is $\left\langle u-\operatorname{proj}_{\mathbb{C}} u, v-\operatorname{proj}_{\mathbb{C}} u\right\rangle \leq 0$ for all $u \in \mathbb{H}, v \in \mathbb{C}$.

In the sequel we will use the following expressions:

(i) $\operatorname{Fix}(\mathbb{T})$ denotes the set of fixed points of $\mathbb{T}$;

(ii) $x_{n} \rightarrow x^{\dagger}$ denotes the weak convergence of $x_{n}$ to $x^{\dagger}$;

(iii) $x_{n} \rightarrow x^{\dagger}$ denotes the strong convergence of $x_{n}$ to $x^{\dagger}$.

The following lemmas will be useful for the next section.

Lemma 2 (see [20]). Let $\mathbb{C}$ be a nonempty closed convex subset of a real Hilbert space $\mathbb{M}$. Let $\mathbb{T}: \mathbb{C} \rightarrow \mathbb{C}$ be a nonexpansive mapping with $\operatorname{Fix}(\mathbb{T}) \neq \emptyset$. Then,

$$
\left\{\begin{array}{c}
\mathbb{C} \supset u_{n} \rightarrow u^{\ddagger} \\
(\mathbb{\square}-\mathbb{T}) u_{n} \longrightarrow v
\end{array}\right\} \Longrightarrow(\mathbb{\square}-\mathbb{T}) u^{\ddagger}=v .
$$

Lemma 3 (see [21]). Let $\mathbb{C}$ be a nonempty closed convex subset of a real Hilbert space $\mathbb{H}$. Assume that a mapping $\mathbb{A}: \mathbb{C} \rightarrow \mathbb{H}$ is monotone and weakly continuous along segments (i.e., $\mathbb{A}(x+$ ty) $\rightarrow \mathbb{A}(x)$ weakly, as $t \rightarrow 0$, whenever $x+t y \in \mathbb{C}$ for $x, y \in \mathbb{C})$. Then the variational inequality

$$
x^{\ddagger} \in C, \quad\left\langle\mathbb{A} x^{\ddagger}, x-x^{\ddagger}\right\rangle \geq 0, \quad \forall x \in \mathbb{C},
$$

is equivalent to the dual variational inequality

$$
x^{\ddagger} \in \mathbb{C}, \quad\left\langle\mathbb{A} x, x-x^{\ddagger}\right\rangle \geq 0, \quad \forall x \in \mathbb{C} .
$$

Lemma 4 (see [22]). Assume that the sequence $\left\{a_{n}\right\}$ satisfies $a_{n} \geq 0$ and $a_{n+1} \leq\left(1-v_{n}\right) a_{n}+\varsigma_{n} v_{n}$ where $\left\{v_{n}\right\}$ is a sequence in $(0,1)$ and $\left\{s_{n}\right\}$ is a sequence such that $\sum_{n=1}^{\infty} \nu_{n}=\infty$ and $\lim \sup _{n \rightarrow \infty} \varsigma_{n} \leq 0$ (or $\left.\sum_{n=1}^{\infty}\left|\varsigma_{n} v_{n}\right|<\infty\right)$. Then $\lim _{n \rightarrow \infty} a_{n}=$ 0 .

\section{Main Results}

In order to prove our main result, we need the following proposition.

Proposition 5. Let $\mathbb{C}$ be a nonempty closed convex subset of a real Hilbert space $\mathbb{H}$. Let $\mathbb{W}: \mathbb{C} \rightarrow \mathbb{C}$ be a nonexpansive mapping with $\operatorname{Fix}(\mathbb{W}) \neq \emptyset$. Let $\varrho: \mathbb{C} \rightarrow \mathbb{U}$ be a $\kappa$-contraction.

For each $t \in(0,1)$, let the net $\left\{u_{t}\right\}$ be defined by

$$
u_{t}=\mathbb{W} \operatorname{proj}_{\mathbb{C}}\left[t \varrho\left(u_{t}\right)+(1-t) u_{t}\right]
$$


Then, as $t \rightarrow 0^{+}$, the net $\left\{u_{t}\right\}$ converges strongly to a point $x^{\ddagger} \in \operatorname{Fix}(\mathbb{W})$ which solves the following variational inequality:

$$
x^{\ddagger} \in \operatorname{Fix}(\mathbb{W}), \quad\left\langle(I-\varrho) x^{\ddagger}, z-x^{\ddagger}\right\rangle \geq 0, \quad z \in \operatorname{Fix}(\mathbb{W}) \text {. }
$$

Proof. For $t \in(0,1)$, define a mapping $\mathbb{W}_{t}: \mathbb{C} \rightarrow \mathbb{C}$ by

$$
\mathbb{W}_{t} u=\mathbb{W} \operatorname{proj}_{\mathbb{C}}[t \varrho(u)+(1-t) u], \quad u \in \mathbb{C} .
$$

For any $u, v \in \mathbb{C}$, we have

$$
\begin{aligned}
\left\|\mathbb{W}_{t} u-\mathbb{W}_{t} v\right\|= & \| \mathbb{W} \operatorname{proj}_{\mathbb{C}}[t \varrho(u)+(1-t) u] \\
& \quad-\mathbb{W} \operatorname{proj}_{\mathbb{C}}[t \varrho(v)+(1-t) v] \| \\
\leq & t\|\varrho(u)-\varrho(v)\|+(1-t)\|u-v\| \\
\leq & {[1-(1-\kappa) t]\|u-v\| . }
\end{aligned}
$$

Hence, $\mathbb{W}_{t}$ is a $1-(1-\kappa) t$-contraction on $\mathbb{C}$ with $u_{t} \in \mathbb{C}$ as its unique fixed point. So, $\left\{u_{t}\right\}$ is well defined.

Let $u \in \operatorname{Fix}(\mathbb{W})$. From (9), we have

$$
\begin{aligned}
\left\|u_{t}-u\right\| & =\left\|\mathbb{W} \operatorname{proj}_{\mathbb{C}}\left[t \varrho\left(u_{t}\right)+(1-t) u_{t}\right]-\mathbb{W} \operatorname{proj}_{\mathbb{C}} u\right\| \\
& \leq t\left\|\varrho\left(u_{t}\right)-\varrho(u)\right\|+t\|\varrho(u)-u\|+(1-t)\left\|u_{t}-u\right\| \\
& \leq[1-(1-\kappa) t]\left\|u_{t}-u\right\|+t\|\varrho(u)-u\| .
\end{aligned}
$$

It follows that

$$
\left\|u_{t}-u\right\| \leq \frac{\|\varrho(u)-u\|}{1-\kappa} .
$$

Thus, $\left\{u_{t}\right\}$ and $\left\{\varrho\left(u_{t}\right)\right\}$ are bounded.

Again from (9), we get

$$
\begin{aligned}
\left\|u_{t}-\mathbb{W} u_{t}\right\| & =\left\|\mathbb{W} \operatorname{proj}_{\mathbb{C}}\left[t \varrho\left(u_{t}\right)+(1-t) u_{t}\right]-\mathbb{W} \operatorname{proj}_{\mathbb{C}} u_{t}\right\| \\
& \leq t\left\|\varrho\left(u_{t}\right)-u_{t}\right\| \longrightarrow 0 \text { as } t \longrightarrow 0^{+} .
\end{aligned}
$$

Let $\left\{t_{n}\right\} \subset(0,1)$ be a sequence such that $t_{n} \rightarrow 0^{+}$as $n \rightarrow \infty$. Put $u_{n}:=u_{t_{n}}$. From (15), we have

$$
\left\|u_{n}-\mathbb{W} u_{n}\right\| \longrightarrow 0 .
$$

From (9), we obtain

$$
\begin{aligned}
\left\|u_{t}-u\right\|^{2}= & \left\|\mathbb{W} \operatorname{proj}_{\mathbb{C}}\left[t \varrho\left(u_{t}\right)+(1-t) u_{t}\right]-\mathbb{W} \operatorname{proj}_{\mathbb{C}} u\right\|^{2} \\
\leq & \left\|u_{t}-u+t\left(\varrho\left(u_{t}\right)-u_{t}\right)\right\|^{2} \\
= & \left\|u_{t}-u\right\|^{2}+2 t\left\langle\varrho\left(u_{t}\right)-u_{t}, u_{t}-u\right\rangle \\
& +t^{2}\left\|\varrho\left(u_{t}\right)-u_{t}\right\|^{2} \\
= & \left\|u_{t}-u\right\|^{2}+2 t\left\langle\varrho\left(u_{t}\right)-\varrho(u), u_{t}-u\right\rangle \\
& +2 t\left\langle\varrho(u)-u, u_{t}-u\right\rangle \\
& +2 t\left\langle u-u_{t}, u_{t}-u\right\rangle+t^{2}\left\|\varrho\left(u_{t}\right)-u_{t}\right\|^{2} \\
\leq & {[1-2(1-\kappa) t]\left\|u_{t}-u\right\|^{2} } \\
& +2 t\left\langle\varrho(u)-u, u_{t}-u\right\rangle+t^{2}\left\|\varrho\left(u_{t}\right)-u_{t}\right\|^{2} .
\end{aligned}
$$

It follows that

$$
\left\|u_{t}-u\right\|^{2} \leq \frac{1}{1-\kappa}\left\langle\varrho(u)-u, u_{t}-u\right\rangle+t M,
$$

where $M>0$ is a constant such that

$$
M>\frac{1}{2(1-\kappa)} \sup \left\{\left\|\varrho\left(u_{t}\right)-u_{t}\right\|^{2}: t \in(0,1)\right\} .
$$

In particular, we have

$$
\left\|u_{n}-u\right\|^{2} \leq \frac{1}{1-\kappa}\left\langle\varrho(u)-u, u_{n}-u\right\rangle+t_{n} M, \quad u \in \operatorname{Fix}(\mathbb{W}) .
$$

Noting that $\left\{u_{n}\right\}$ is bounded, without loss of generality, we assume that $u_{n} \rightarrow x^{\ddagger}$. It is obvious that $x^{\ddagger} \in \mathbb{C}$. From (16) and Lemma 2, we deduce $x^{\ddagger} \in \operatorname{Fix}(\mathbb{W})$. Substitute $x^{\ddagger}$ for $u$ in (20) to get

$$
\left\|u_{n}-x^{\ddagger}\right\|^{2} \leq \frac{1}{1-\kappa}\left\langle\varrho\left(x^{\ddagger}\right)-x^{\ddagger}, x_{n}-x^{\ddagger}\right\rangle+t_{n} M .
$$

Since $u_{n} \rightarrow x^{\ddagger}$, we deduce from (21) that $u_{n} \rightarrow x^{\ddagger}$. The net $\left\{u_{t}\right\}$ is, therefore, relatively compact, as $t \rightarrow 0^{+}$, in the norm topology.

In (20), letting $n \rightarrow \infty$, we get

$$
\left\|x^{\ddagger}-u\right\|^{2} \leq \frac{1}{1-\kappa}\left\langle\varrho(u)-u, x^{\ddagger}-u\right\rangle, \quad u \in \operatorname{Fix}(\mathbb{W}) .
$$

Therefore, $x^{\ddagger}$ solves the variational inequality

$$
x^{\ddagger} \in \operatorname{Fix}(\mathbb{W}), \quad\left\langle(I-\varrho) u, u-x^{\ddagger}\right\rangle \geq 0, \quad u \in \operatorname{Fix}(\mathbb{W}) \text {. }
$$

By Lemma 3, (23) equals its dual variational inequality

$$
x^{\ddagger} \in \operatorname{Fix}(\mathbb{W}), \quad\left\langle(\square-\varrho) x^{\ddagger}, u-x^{\ddagger}\right\rangle \geq 0, \quad u \in \operatorname{Fix}(\mathbb{W}) .
$$

This indicates that $x^{\ddagger}=\left(\operatorname{proj}_{\text {Fix }(\mathbb{W})} \varrho\right) x^{\ddagger}$. That is, $x^{\ddagger}$ is the unique fixed point in $\operatorname{Fix}(\mathbb{W})$ of the contraction $\operatorname{proj}_{\text {Fix }(\mathbb{W})} \varrho$. So, the entire net $\left\{u_{t}\right\}$ converges in norm to $x^{\ddagger}$ as $t \rightarrow 0^{+}$. This completes the proof.

Corollary 6. Let $\mathbb{C}$ be a nonempty closed convex subset of a real Hilbert space $\mathbb{H}$. Let $\mathbb{W}: \mathbb{C} \rightarrow \mathbb{C}$ be a nonexpansive mapping with $\operatorname{Fix}(\mathbb{W}) \neq \emptyset$.

For each $t \in(0,1)$, let the net $\left\{u_{t}\right\}$ be defined by

$$
u_{t}=\mathbb{W} \operatorname{proj}_{\mathbb{C}}\left[(1-t) u_{t}\right]
$$

Then, as $t \rightarrow 0^{+}$, the net $\left\{u_{t}\right\}$ converges strongly to the minimum-norm fixed point of $\mathbb{W}$.

Proof. As a matter of fact, in (9), we choose $\varrho=0$, and then (9) reduces to (25). From Proposition 5, (24) is reduced to

$$
0 \leq\left\langle x^{\ddagger}, u-x^{\ddagger}\right\rangle, \quad u \in \operatorname{Fix}(\mathbb{W}) \text {. }
$$


Equivalently,

$$
\left\|x^{\ddagger}\right\|^{2} \leq\left\langle x^{\ddagger}, u\right\rangle, \quad u \in \operatorname{Fix}(\mathbb{W}) .
$$

This implies that

$$
\left\|x^{\ddagger}\right\| \leq\|u\|, \quad u \in \operatorname{Fix}(\mathbb{W}) .
$$

Therefore, $x^{\ddagger}$ is the minimum-norm fixed point of $\mathbb{W}$. This completes the proof.

Algorithm 7. Let $\mathbb{C}$ be a nonempty closed subset of a real Hilbert space $\mathbb{H}$. Let $\mathbb{T}: \mathbb{C} \rightarrow \mathbb{C}$ be a pseudocontraction

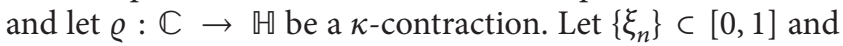
$\left\{\eta_{n}\right\} \subset[0,1]$ be two real number sequences. For $x_{0} \in \mathbb{C}$, we define a sequence $\left\{x_{n}\right\}$ iteratively by

$$
x_{n+1}=\operatorname{proj}_{\mathbb{C}}\left[\xi_{n} \varrho\left(x_{n}\right)+\left(1-\xi_{n}-\eta_{n}\right) x_{n}+\eta_{n} \llbracket x_{n}\right], \quad n \geq 0 .
$$

Theorem 8. Let $\mathbb{C}$ be a nonempty closed convex subset of a real Hilbert space $\mathbb{\square}$. Let $\mathbb{T}: \mathbb{C} \rightarrow \mathbb{C}$ be an L-Lipschitzian and pseudocontraction with $\operatorname{Fix}(\mathbb{T}) \neq \emptyset$ and $\varrho: \mathbb{C} \rightarrow \mathbb{Q} a \kappa$ contraction. Suppose the following conditions are satisfied:

$$
\begin{aligned}
& \text { (C1) } \lim _{n \rightarrow \infty} \xi_{n}=0 \text { and } \sum_{n=0}^{\infty} \xi_{n}=\infty ; \\
& \text { (C2) } \lim _{n \rightarrow \infty}\left(\xi_{n} / \eta_{n}\right)=\lim _{n \rightarrow \infty}\left(\eta_{n}^{2} / \xi_{n}\right)=0 ; \\
& \text { (C3) } \lim _{n \rightarrow \infty}\left(\left(\xi_{n} \eta_{n-1}-\xi_{n-1} \eta_{n}\right) / \xi_{n}^{2} \eta_{n-1}\right)=0 .
\end{aligned}
$$

Then the sequence $\left\{x_{n}\right\}$ generated by the algorithm (29) converges strongly to $x^{\ddagger}=\left(\operatorname{proj}_{\mathrm{Fix}(\mathbb{T})} Q\right) x^{\ddagger}$.

Proof. First, we prove that the sequence $\left\{x_{n}\right\}$ is bounded. We will show this fact by induction. According to conditions (C1) and (C2), there exists a sufficiently large positive integer $m$ such that

$$
1-\frac{2}{1-\kappa}(L+1)(L+3)\left(\xi_{n}+2 \eta_{n}+\frac{\eta_{n}^{2}}{\xi_{n}}\right)>0, \quad n \geq m .
$$

Fix a $p \in \operatorname{Fix}(\mathbb{T})$ and take a constant $M_{1}>0$ such that

$$
\begin{aligned}
\max & \left\{\left\|x_{0}-p\right\|,\left\|x_{1}-p\right\|, \ldots,\left\|x_{m}-p\right\|, 2\|\varrho(p)-p\|\right\} \\
& \leq M_{1} .
\end{aligned}
$$

Next, we show that $\left\|x_{m+1}-p\right\| \leq M_{1}$.

Set

$$
\begin{aligned}
& y_{m}=\xi_{m} \varrho\left(x_{m}\right)+\left(1-\xi_{m}-\eta_{m}\right) x_{m}+\eta_{m} \rrbracket x_{m} ; \\
& \text { thus, } x_{m+1}=\operatorname{proj}_{\mathbb{C}}\left[y_{m}\right] \text {. }
\end{aligned}
$$

Then we have

$$
\left\langle x_{m+1}-y_{m}, x_{m+1}-p\right\rangle \leq 0 .
$$

Since $\mathbb{T}$ is pseudocontractive, $\mathbb{\square}-\mathbb{T}$ is monotone. So, we have

$$
\left\langle(\mathbb{\square}-\mathbb{T}) x_{m+1}-(\mathbb{\square}-\mathbb{T}) p, x_{m+1}-p\right\rangle \geq 0 .
$$

From (29), (33), and (34), we obtain

$$
\begin{aligned}
& \left\|x_{m+1}-p\right\|^{2}=\left\langle x_{m+1}-p, x_{m+1}-p\right\rangle \\
& =\left\langle x_{m+1}-y_{m}, x_{m+1}-p\right\rangle+\left\langle y_{m}-p, x_{m+1}-p\right\rangle \\
& \leq\left\langle y_{m}-p, x_{m+1}-p\right\rangle \\
& =\left\langle x_{m}-p, x_{m+1}-p\right\rangle \\
& -\xi_{m}\left\langle x_{m}-\varrho\left(x_{m}\right), x_{m+1}-p\right\rangle \\
& +\eta_{m}\left\langle\mathbb{T} x_{m}-x_{m}, x_{m+1}-p\right\rangle \\
& =\left\langle x_{m}-p, x_{m+1}-p\right\rangle \\
& +\xi_{m}\left\langle x_{m+1}-x_{m}, x_{m+1}-p\right\rangle \\
& +\xi_{m}\left\langle\varrho\left(x_{m}\right)-\varrho(p), x_{m+1}-p\right\rangle \\
& +\xi_{m}\left\langle f(p)-p, x_{m+1}-p\right\rangle \\
& -\xi_{m}\left\langle x_{m+1}-p, x_{m+1}-p\right\rangle \\
& +\eta_{m}\left\langle\mathbb{T} x_{m}-\mathbb{T} x_{m+1}, x_{m+1}-p\right\rangle \\
& +\eta_{m}\left\langle x_{m+1}-x_{m}, x_{m+1}-p\right\rangle \\
& -\eta_{m}\left\langle x_{m+1}-\mathbb{T} x_{m+1}, x_{m+1}-p\right\rangle \\
& \leq\left\|x_{m}-p\right\|\left\|x_{m+1}-p\right\|+\xi_{m}\left\|x_{m+1}-x_{m}\right\| \\
& \times\left\|x_{m+1}-p\right\|+\xi_{m} \kappa\left\|x_{m}-p\right\|\left\|x_{m+1}-p\right\| \\
& +\xi_{m}\|\varrho(p)-p\|\left\|x_{m+1}-p\right\|-\xi_{m}\left\|x_{m+1}-p\right\|^{2} \\
& +\eta_{m}\left(\left\|\mathbb{\top} x_{m}-\mathbb{T} x_{m+1}\right\|+\left\|x_{m+1}-x_{m}\right\|\right) \\
& \times\left\|x_{m+1}-p\right\| \\
& \leq\left(1+\xi_{m} \kappa\right)\left\|x_{m}-p\right\| \\
& \times\left\|x_{m+1}-p\right\|+\xi_{m}\|\varrho(p)-p\| \\
& \times\left\|x_{m+1}-p\right\|-\xi_{m}\left\|x_{m+1}-p\right\|^{2} \\
& +(L+1)\left(\xi_{m}+\eta_{m}\right)\left\|x_{m+1}-x_{m}\right\|\left\|x_{m+1}-p\right\| .
\end{aligned}
$$

It follows that

$$
\begin{aligned}
\left(1+\xi_{m}\right)\left\|x_{m+1}-p\right\| \leq & \left(1+\xi_{m} \kappa\right)\left\|x_{m}-p\right\| \\
& +\xi_{m}\|\varrho(p)-p\| \\
& +(L+1)\left(\xi_{m}+\eta_{m}\right)\left\|x_{m+1}-x_{m}\right\| .
\end{aligned}
$$


By (29), we have

$$
\begin{aligned}
& \left\|x_{m+1}-x_{m}\right\| \\
& =\| \operatorname{proj}_{\mathbb{C}}\left[\xi_{m} \varrho\left(x_{m}\right)+\left(1-\xi_{m}-\eta_{m}\right) x_{m}+\eta_{m} \rrbracket x_{m}\right] \\
& -\operatorname{proj}_{\mathbb{C}}\left[x_{m}\right] \| \\
& \leq\left\|\xi_{m} \varrho\left(x_{m}\right)+\left(1-\xi_{m}-\eta_{m}\right) x_{m}+\eta_{m} \rrbracket x_{m}-x_{m}\right\| \\
& \leq \xi_{m}\left(\left\|\varrho\left(x_{m}\right)-\varrho(p)\right\|+\|\varrho(p)-p\|+\left\|x_{m}-p\right\|\right) \\
& +\eta_{m}\left(\left\|\rrbracket x_{m}-p\right\|+\left\|x_{m}-p\right\|\right) \\
& \leq \xi_{m}\left(\|\varrho(p)-p\|+(1+\kappa)\left\|x_{m}-p\right\|\right) \\
& +(L+1) \eta_{m}\left\|x_{m}-p\right\| \\
& \leq(L+1+\kappa)\left(\xi_{m}+\eta_{m}\right)\left\|x_{m}-p\right\|+\xi_{m}\|\varrho(p)-p\| \\
& \leq(L+3)\left(\xi_{m}+\eta_{m}\right) M_{1} \text {. }
\end{aligned}
$$

Substitute (37) into (36) to obtain

$$
\begin{aligned}
\left(1+\xi_{m}\right) & \left\|x_{m+1}-p\right\| \\
\leq & \left(1+\xi_{m} \kappa\right)\left\|x_{m}-p\right\|+\xi_{m}\|\varrho(p)-p\| \\
& +(L+1)(L+3)\left(\xi_{m}+\eta_{m}\right)^{2} M_{1} \\
& \leq\left(1+\frac{1+\kappa}{2} \xi_{m}\right) M_{1}+(L+1)(L+3)\left(\xi_{m}+\eta_{m}\right)^{2} M_{1}
\end{aligned}
$$

that is,

$$
\begin{aligned}
& \left\|x_{m+1}-p\right\| \\
& \leq\left[1-\frac{\left((1-\kappa) \xi_{m} / 2\right)-(L+1)(L+3)\left(\xi_{m}+\eta_{m}\right)^{2}}{1+\xi_{m}}\right] M_{1} \\
& =\left\{1-\left(\left(( \frac { ( 1 - \kappa ) \xi _ { m } } { 2 } ) \left[1-\frac{2}{1-\kappa}(L+1)(L+3)\right.\right.\right.\right. \\
& \left.\left.\times\left(\xi_{m}+2 \eta_{m}+\left(\frac{\eta_{m}^{2}}{\xi_{m}}\right)\right)\right]\right) \\
& \left.\left.\times\left(1+\xi_{m}\right)^{-1}\right)\right\} M_{1} \\
& \leq M_{1} .
\end{aligned}
$$

By induction, we get

$$
\left\|x_{n}-p\right\| \leq M_{1}, \quad \forall n \geq 0,
$$

which implies that $\left\{x_{n}\right\}$ is bounded and so is $\left\{\llbracket x_{n}\right\}$. Now, we take a constant $M_{2}>0$ such that

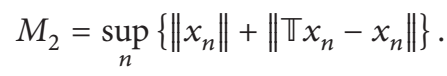

Set $\mathbb{S}=(2 \rrbracket-\mathbb{T})^{-1}$ (i.e., $\mathbb{S}$ is a resolvent of the monotone operator $\square-\mathbb{T})$. We then have that $\mathbb{S}$ is a nonexpansive self mapping of $\mathbb{C}$ and $\operatorname{Fix}(\mathbb{S})=\operatorname{Fix}(\mathbb{T})$.

By Proposition 5, we know that, whenever $\left\{\gamma_{n}\right\} \subset(0,1)$ and $\gamma_{n} \rightarrow 0^{+}$, the sequence $\left\{z_{n}\right\}$ defined by

$$
z_{n}=\mathbb{S} \operatorname{proj}_{\mathbb{C}}\left[\gamma_{n} \varrho\left(z_{n}\right)+\left(1-\gamma_{n}\right) z_{n}\right]
$$

converges strongly to the fixed point $x^{\ddagger}$ of $\mathbb{S}$ (and of $\mathbb{T}$ as $\operatorname{Fix}(\mathbb{S})=\operatorname{Fix}(\mathbb{T}))$. Without loss of generality, we may assume that $\left\|z_{n}\right\| \leq M_{2}$ for all $n$.

It suffices to prove that $\left\|x_{n+1}-z_{n}\right\| \rightarrow 0$ as $n \rightarrow \infty$ (for some $\gamma_{n} \rightarrow 0^{+}$). To this end, we rewrite (42) as

$$
(2 \llbracket-\mathbb{T}) z_{n}=\operatorname{proj}_{\mathbb{C}}\left[\gamma_{n} \varrho\left(z_{n}\right)+\left(1-\gamma_{n}\right) z_{n}\right], \quad n \geq 0 .
$$

By using the property of the metric projection, we have

$$
\begin{aligned}
& \left\langle\gamma_{n} \varrho\left(z_{n}\right)+\left(1-\gamma_{n}\right) z_{n}-\left(2 z_{n}-\mathbb{\nabla} z_{n}\right), x_{n+1}\right. \\
& \left.-\left(2 z_{n}-\mathbb{} z_{n}\right)\right\rangle \leq 0 \\
& \Longrightarrow\left\langle-\gamma_{n}\left(z_{n}-\varrho\left(z_{n}\right)\right), x_{n+1}-z_{n}-\left(z_{n}-\mathbb{} z_{n}\right)\right\rangle \\
& +\left\langle\llbracket z_{n}-z_{n}, x_{n+1}-z_{n}-\left(z_{n}-\llbracket z_{n}\right)\right\rangle \leq 0 \\
& \Longrightarrow\left\langle-\gamma_{n}\left(z_{n}-\varrho\left(z_{n}\right)\right)+\mathbb{\nabla} z_{n}-z_{n}, x_{n+1}-z_{n}\right\rangle \\
& +\left\|z_{n}-\mathbb{\nabla} z_{n}\right\|^{2} \leq\left\langle\gamma_{n}\left(z_{n}-\varrho\left(z_{n}\right)\right), \mathbb{} z_{n}-z_{n}\right\rangle \\
& \Longrightarrow\left\langle-\gamma_{n}\left(z_{n}-\varrho\left(z_{n}\right)\right)+\mathbb{\top} z_{n}-z_{n}, x_{n+1}-z_{n}\right\rangle
\end{aligned}
$$

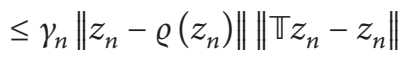

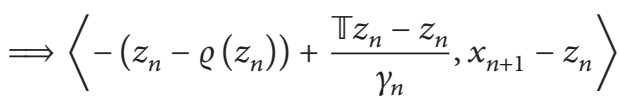

$$
\begin{aligned}
& \leq\left\|z_{n}-\varrho\left(z_{n}\right)\right\|\left\|\mathbb{T} z_{n}-z_{n}\right\| .
\end{aligned}
$$

Note that

$$
\begin{aligned}
\left\|z_{n}-\mathbb{T} z_{n}\right\| & =\left\|\operatorname{proj}_{\mathbb{C}}\left[\gamma_{n} \varrho\left(z_{n}\right)+\left(1-\gamma_{n}\right) z_{n}\right]-z_{n}\right\| \\
& \leq\left\|\gamma_{n} \varrho\left(z_{n}\right)+\left(1-\gamma_{n}\right) z_{n}-z_{n}\right\| \\
& =\gamma_{n}\left\|z_{n}-\varrho\left(z_{n}\right)\right\| .
\end{aligned}
$$

Hence, we get

$$
\left\langle-\left(z_{n}-\varrho\left(z_{n}\right)\right)+\frac{\mathbb{\varpi} z_{n}-z_{n}}{\gamma_{n}}, x_{n+1}-z_{n}\right\rangle \leq \gamma_{n}\left\|z_{n}-\varrho\left(z_{n}\right)\right\|^{2} .
$$


From (42), we have

$$
\begin{aligned}
\left\|z_{n+1}-z_{n}\right\|= & \| \mathbb{S}_{\operatorname{proj}_{\mathbb{C}}}\left[\gamma_{n+1} \varrho\left(z_{n+1}\right)+\left(1-\gamma_{n+1}\right) z_{n+1}\right] \\
& -\mathbb{S}_{\operatorname{proj}_{\mathbb{C}}}\left[\gamma_{n} \varrho\left(z_{n}\right)+\left(1-\gamma_{n}\right) z_{n}\right] \| \\
\leq & \| \gamma_{n+1} \varrho\left(z_{n+1}\right)+\left(1-\gamma_{n+1}\right) z_{n+1} \\
\quad & -\gamma_{n} \varrho\left(z_{n}\right)-\left(1-\gamma_{n}\right) z_{n} \| \\
= & \|\left(1-\gamma_{n+1}\right)\left(z_{n+1}-z_{n}\right)+\left(\gamma_{n}-\gamma_{n+1}\right) \\
& \times\left(z_{n}-\varrho\left(z_{n}\right)\right)+\gamma_{n+1}\left(\varrho\left(z_{n+1}\right)-\varrho\left(z_{n}\right)\right) \| \\
\leq & {\left[1-(1-\kappa) \gamma_{n+1}\right]\left\|z_{n+1}-z_{n}\right\| } \\
& +\left|\gamma_{n+1}-\gamma_{n}\right|\left\|z_{n}-\varrho\left(z_{n}\right)\right\| .
\end{aligned}
$$

It follows that

$$
\left\|z_{n+1}-z_{n}\right\| \leq \frac{\left|\gamma_{n+1}-\gamma_{n}\right|}{(1-\kappa) \gamma_{n+1}}\left\|z_{n}-\varrho\left(z_{n}\right)\right\| .
$$

Set

$$
\gamma_{n}:=\frac{\xi_{n}}{\eta_{n}}
$$

By condition (C2), $\gamma_{n} \rightarrow 0^{+}$and $\gamma_{n} \in(0,1)$, for $n$ large enough. Hence, by (46) and (48), we have

$$
\begin{gathered}
\left\langle-\left(z_{n}-\varrho\left(z_{n}\right)\right)+\frac{\eta_{n}\left(\mathbb{\pi} z_{n}-z_{n}\right)}{\xi_{n}}, x_{n+1}-z_{n}\right\rangle \\
\leq \frac{\xi_{n}}{\eta_{n}}\left\|z_{n}-\varrho\left(z_{n}\right)\right\|^{2} \leq \frac{\xi_{n}}{\eta_{n}} M_{2}^{2}, \\
\left\|z_{n}-z_{n-1}\right\| \leq \frac{\left|\xi_{n} \eta_{n-1}-\xi_{n-1} \eta_{n}\right|}{\xi_{n} \eta_{n-1}} M_{2} .
\end{gathered}
$$

By (29), we have

$$
\begin{aligned}
& \left\|x_{n+1}-x_{n}\right\| \\
& =\| \operatorname{proj}_{\mathbb{C}}\left[\xi_{n} \varrho\left(x_{n}\right)+\left(1-\xi_{n}-\eta_{n}\right) x_{n}+\eta_{n} \mathbb{T} x_{n}\right] \\
& -\operatorname{proj}_{\mathbb{C}} x_{n} \| \\
& \leq \xi_{n}\left\|x_{n}-\varrho\left(x_{n}\right)\right\|+\eta_{n}\left\|\mathbb{\top} x_{n}-x_{n}\right\| \\
& \leq\left(\xi_{n}+\eta_{n}\right) M_{2} \text {. }
\end{aligned}
$$

Next, we estimate $\left\|x_{n+1}-z_{n+1}\right\|$. Since $x_{n+1}=\operatorname{proj}_{\mathbb{C}}\left[y_{n}\right]$, $\left\langle x_{n+1}-y_{n}, x_{n+1}-z_{n}\right\rangle \leq 0$. Then, we have

$$
\begin{aligned}
\left\|x_{n+1}-z_{n}\right\|^{2}= & \left\langle x_{n+1}-z_{n}, x_{n+1}-z_{n}\right\rangle \\
= & \left\langle x_{n+1}-y_{n}, x_{n+1}-z_{n}\right\rangle \\
& +\left\langle y_{n}-z_{n}, x_{n+1}-z_{n}\right\rangle \\
\leq & \left\langle y_{n}-z_{n}, x_{n+1}-z_{n}\right\rangle \\
= & \left\langle\left[\xi_{n} \varrho\left(x_{n}\right)+\left(1-\xi_{n}-\eta_{n}\right) x_{n}+\eta_{n} \mathbb{T} x_{n}\right]\right. \\
& \left.\quad-z_{n}, x_{n+1}-z_{n}\right\rangle
\end{aligned}
$$

$$
\begin{aligned}
& =\left(1-\xi_{n}-\eta_{n}\right)\left\langle x_{n}-z_{n}, x_{n+1}-z_{n}\right\rangle
\end{aligned}
$$

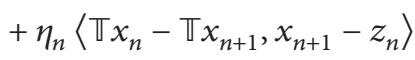

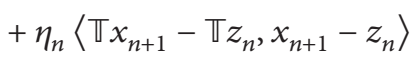

$$
\begin{aligned}
& +\left\langle\xi_{n}\left(\varrho\left(x_{n}\right)-z_{n}\right)\right. \\
& \left.+\eta_{n}\left(\mathbb{} z_{n}-z_{n}\right), x_{n+1}-z_{n}\right\rangle .
\end{aligned}
$$

It follows that

$$
\begin{aligned}
\| x_{n+1} & -z_{n} \|^{2} \\
\leq & \left(1-\xi_{n}-\eta_{n}\right)\left\|x_{n}-z_{n}\right\|\left\|x_{n+1}-z_{n}\right\| \\
& +\eta_{n} L\left\|x_{n}-x_{n+1}\right\|\left\|x_{n+1}-z_{n}\right\| \\
& +\eta_{n}\left\|x_{n+1}-z_{n}\right\|^{2} \\
& +\xi_{n}\left\langle\varrho\left(z_{n}\right)-z_{n}+\frac{\eta_{n}}{\xi_{n}}\left(\mathbb{V} z_{n}-z_{n}\right), x_{n+1}-z_{n}\right\rangle \\
\leq & \frac{1-\xi_{n}-\eta_{n}}{2}\left(\left\|x_{n}-z_{n}\right\|^{2}+\left\|x_{n+1}-z_{n}\right\|^{2}\right) \\
& +\frac{\eta_{n}^{2}}{2}\left\|x_{n+1}-z_{n}\right\|^{2}+\frac{L^{2}}{2}\left\|x_{n}-x_{n+1}\right\|^{2} \\
& +\eta_{n}\left\|x_{n+1}-z_{n}\right\|^{2}+\frac{\xi_{n}^{2}}{\eta_{n}}\left\|z_{n}-\varrho\left(z_{n}\right)\right\|^{2} .
\end{aligned}
$$

Thus,

$$
\begin{aligned}
\left\|x_{n+1}-z_{n}\right\|^{2} \leq & \frac{1-\xi_{n}-\eta_{n}}{1+\xi_{n}-\eta_{n}}\left\|x_{n}-z_{n}\right\|^{2} \\
& +\frac{L^{2}}{1+\xi_{n}-\eta_{n}}\left\|x_{n+1}-x_{n}\right\|^{2} \\
& +\frac{2 \xi_{n}^{2}}{\left(1+\xi_{n}-\eta_{n}\right) \eta_{n}}\left\|z_{n}-\varrho\left(z_{n}\right)\right\|^{2} \\
& +\frac{\eta_{n}^{2}}{1+\xi_{n}-\eta_{n}}\left\|x_{n+1}-z_{n}\right\|^{2} \\
\leq & \left(1-\frac{2 \xi_{n}}{1+\xi_{n}-\eta_{n}}\right)\left\|x_{n}-z_{n}\right\|^{2} \\
& +\frac{\left(\xi_{n}+\eta_{n}\right)^{2}}{1+\xi_{n}-\eta_{n}} L^{2} M_{2}^{2}+\frac{2 \xi_{n}^{2}}{\left(1+\xi_{n}-\eta_{n}\right) \eta_{n}} M_{2}^{2} \\
& +\frac{\eta_{n}^{2}}{1+\xi_{n}-\eta_{n}} 4 M_{2}^{2} \\
\leq & \left(1-\frac{2 \xi_{n}}{1+\xi_{n}-\eta_{n}}\right) \\
& \times\left(\left\|x_{n}-z_{n-1}\right\|+\left\|z_{n}-z_{n-1}\right\|\right)^{2} \\
& \left(1-\frac{1}{1-1}\right)
\end{aligned}
$$




$$
\begin{aligned}
& +\left\{\frac{\left(\xi_{n}+\eta_{n}\right)^{2}}{1+\xi_{n}-\eta_{n}}+\frac{2 \xi_{n}^{2}}{\left(1+\xi_{n}-\eta_{n}\right) \eta_{n}}\right. \\
& \left.+\frac{\eta_{n}^{2}}{1+\xi_{n}-\eta_{n}}\right\} M \\
& \leq\left(1-\frac{2 \xi_{n}}{1+\xi_{n}-\eta_{n}}\right)\left\|x_{n}-z_{n-1}\right\|^{2} \\
& +\frac{1}{1+\xi_{n}-\eta_{n}}\left\|z_{n}-z_{n-1}\right\| \\
& \times\left(2\left\|x_{n}-z_{n-1}\right\|+\left\|z_{n}-z_{n-1}\right\|\right) \\
& +\left\{\frac{\left(\xi_{n}+\eta_{n}\right)^{2}}{1+\xi_{n}-\eta_{n}}+\frac{2 \xi_{n}^{2}}{\left(1+\xi_{n}-\eta_{n}\right) \eta_{n}}\right. \\
& \left.+\frac{\eta_{n}^{2}}{1+\xi_{n}-\eta_{n}}\right\} M \\
& \leq\left(1-\frac{2 \xi_{n}}{1+\xi_{n}-\eta_{n}}\right)\left\|x_{n}-z_{n-1}\right\|^{2} \\
& +\frac{1}{1+\xi_{n}-\eta_{n}} \frac{\left|\xi_{n} \eta_{n-1}-\xi_{n-1} \eta_{n}\right|}{\xi_{n} \eta_{n-1}} M \\
& +\left\{\frac{\left(\xi_{n}+\eta_{n}\right)^{2}}{1+\xi_{n}-\eta_{n}}+\frac{2 \xi_{n}^{2}}{\left(1+\xi_{n}-\eta_{n}\right) \eta_{n}}\right. \\
& \left.+\frac{\eta_{n}^{2}}{1+\xi_{n}-\eta_{n}}\right\} M
\end{aligned}
$$

where the finite constant $M>0$ is given by

$$
\begin{aligned}
M:=\max \{ & L^{2} M_{2}^{2}, 4 M_{2}^{2}, \\
& \left.M_{2} \sup _{n}\left(2\left\|x_{n}-z_{n-1}\right\|+\left\|z_{n}-z_{n-1}\right\|\right)\right\} .
\end{aligned}
$$

Set

$$
\begin{gathered}
\delta_{n}=\frac{2 \xi_{n}}{1+\xi_{n}-\eta_{n}} \approx 2 \xi_{n} \quad(\text { as } n \longrightarrow \infty), \\
\theta_{n}=\left\{\frac{\xi_{n} \eta_{n-1}-\xi_{n-1} \eta_{n}}{2 \xi_{n}^{2} \eta_{n-1}}\right. \\
\left.+\frac{1}{2}\left(\xi_{n}+2 \eta_{n}+\frac{\eta_{n}^{2}}{\xi_{n}}\right)+\frac{\xi_{n}}{\eta_{n}}+\frac{\eta_{n}^{2}}{2 \xi_{n}}\right\} M .
\end{gathered}
$$

Then (54) can be rewritten as

$$
\left\|x_{n+1}-z_{n}\right\|^{2} \leq\left(1-\delta_{n}\right)\left\|x_{n}-z_{n-1}\right\|^{2}+\delta_{n} \theta_{n} .
$$

By the conditions $(\mathrm{C} 1)-(\mathrm{C} 3)$, we deuce that

$$
\lim _{n \rightarrow \infty} \delta_{n}=0, \quad \sum_{n=1}^{\infty} \delta_{n}=\infty, \quad \lim _{n \rightarrow \infty} \theta_{n}=0 .
$$

From Lemma 4 and (57), we get $\left\|x_{n+1}-z_{n}\right\|^{2} \rightarrow 0$ as $n \rightarrow$ $\infty$. This completes the proof.

Algorithm 9. Let $\mathbb{C}$ be a nonempty closed subset of a real Hilbert space $\mathbb{T}$. Let $\mathbb{\mathbb { T }}: \mathbb{C} \rightarrow \mathbb{C}$ be a pseudocontraction. Let $\left\{\xi_{n}\right\} \subset[0,1]$ and $\left\{\eta_{n}\right\} \subset[0,1]$ be two real number sequences. For $x_{0} \in \mathbb{C}$, we define a sequence $\left\{x_{n}\right\}$ iteratively by

$$
x_{n+1}=\operatorname{proj}_{\mathbb{C}}\left[\left(1-\xi_{n}-\eta_{n}\right) x_{n}+\eta_{n} \rrbracket x_{n}\right], \quad n \geq 0 .
$$

Corollary 10. Let $\mathbb{C}$ be a nonempty closed convex subset of a real Hilbert space $\mathbb{H}$. Let $\mathbb{T}: \mathbb{C} \rightarrow \mathbb{C}$ be an L-Lipschitzian and pseudocontraction with $\operatorname{Fix}(\mathbb{T}) \neq \emptyset$. Suppose the following conditions are satisfied:

$$
\begin{aligned}
& \text { (C1) } \lim _{n \rightarrow \infty} \xi_{n}=0 \text { and } \sum_{n=0}^{\infty} \xi_{n}=\infty ; \\
& \text { (C2) } \lim _{n \rightarrow \infty}\left(\xi_{n} / \eta_{n}\right)=\lim _{n \rightarrow \infty}\left(\eta_{n}^{2} / \xi_{n}\right)=0 ; \\
& \text { (C3) } \lim _{n \rightarrow \infty}\left(\left(\xi_{n} \eta_{n-1}-\xi_{n-1} \eta_{n}\right) / \xi_{n}^{2} \eta_{n-1}\right)=0 .
\end{aligned}
$$

Then the sequence $\left\{x_{n}\right\}$ generated by the algorithm (59) converges strongly to the minimum-norm fixed point of $\mathbb{T}$.

\section{Conflict of Interests}

The authors declare that there is no conflict of interests regarding the publication of this paper.

\section{Acknowledgment}

The first author was supported by the National Natural Science Foundation of China, Grant no. 11226125.

\section{References}

[1] K. Deimling, "Zeros of accretive operators," Manuscripta Mathematica, vol. 13, no. 4, pp. 365-374, 1974.

[2] S. Ishikawa, "Fixed points by a new iteration method," Proceedings of the American Mathematical Society, vol. 44, pp. 147-150, 1974.

[3] C. E. Chidume and S. A. Mutangadura, "An example on the mann iteration method for lipschitz pseudocontractions," Proceedings of the American Mathematical Society, vol. 129, no. 8, pp. 2359-2363, 2001.

[4] H. Zhou, "Convergence theorems of fixed points for Lipschitz pseudo-contractions in Hilbert spaces," Journal of Mathematical Analysis and Applications, vol. 343, no. 1, pp. 546-556, 2008.

[5] Y. Yao, Y.-C. Liou, and G. Marino, "A hybrid algorithm for pseudo-contractive mappings," Nonlinear Analysis, Theory, Methods and Applications, vol. 71, no. 10, pp. 4997-5002, 2009.

[6] H. Zegeye, N. Shahzad, and M. A. Alghamdi, "Convergence of Ishikawa's iteration method for pseudocontractive mappings," Nonlinear Analysis, Theory, Methods and Applications, vol. 74, no. 18, pp. 7304-7311, 2011.

[7] L.-C. Ceng, A. Petruşel, and J.-C. Yao, "Strong convergence of modified implicit iterative algorithms with perturbed mappings for continuous pseudocontractive mappings," Applied Mathematics and Computation, vol. 209, no. 2, pp. 162-176, 2009.

[8] L.-C. Ceng, A. Petruşel, and J.-C. Yao, "Iterative approximation of fixed points for asymptotically strict pseudocontractive type mappings in the intermediate sense," Taiwanese Journal of Mathematics, vol. 15, no. 2, pp. 587-606, 2011. 
[9] L.-C. Ceng and J.-C. Yao, "Strong convergence theorems for variational inequalities and fixed point problems of asymptotically strict pseudocontractive mappings in the intermediate sense," Acta Applicandae Mathematicae, vol. 115, no. 2, pp. 167191, 2011.

[10] J. C. Yao and L. C. Zeng, "Strong convergence of averaged approximants for asymptotically pseudocontractive mappings in Banach spaces," Journal of Nonlinear and Convex Analysis, vol. 8, pp. 451-462, 2007.

[11] L. C. Ceng, Q. H. Ansari, and C. F. Wen, "Implicit relaxed and hybrid methods with regularization for minimization problems and asymptotically strict pseudocontractive mappings in the intermediate sense," Abstract and Applied Analysis, vol. 2013, Article ID 854297, 14 pages, 2013.

[12] C. E. Chidume, M. Abbas, and B. Ali, "Convergence of the Mann iteration algorithm for a class of pseudocontractive mappings," Applied Mathematics and Computation, vol. 194, no. 1, pp. 1-6, 2007.

[13] C. E. Chidume and H. Zegeye, "Iterative solution of nonlinear equations of accretive and pseudocontractive types," Journal of Mathematical Analysis and Applications, vol. 282, no. 2, pp. 756765, 2003.

[14] L. Ćirić, A. Rafiq, N. Cakić, and J. S. Ume, "Implicit Mann fixed point iterations for pseudo-contractive mappings," Applied Mathematics Letters, vol. 22, no. 4, pp. 581-584, 2009.

[15] C. Moore and B. V. C. Nnoli, "Strong convergence of averaged approximants for Lipschitz pseudocontractive maps," Journal of Mathematical Analysis and Applications, vol. 260, no. 1, pp. 269278, 2001.

[16] A. Udomene, "Path convergence, approximation of fixed points and variational solutions of Lipschitz pseudocontractions in Banach spaces," Nonlinear Analysis, Theory, Methods and Applications, vol. 67, no. 8, pp. 2403-2414, 2007.

[17] H. Zegeye, N. Shahzad, and T. Mekonen, "Viscosity approximation methods for pseudocontractive mappings in Banach spaces," Applied Mathematics and Computation, vol. 185, no. 1, pp. 538-546, 2007.

[18] Q.-B. Zhang and C.-Z. Cheng, "Strong convergence theorem for a family of Lipschitz pseudocontractive mappings in a Hilbert space," Mathematical and Computer Modelling, vol. 48, no. 3-4, pp. 480-485, 2008.

[19] H. Zhou, "Strong convergence of an explicit iterative algorithm for continuous pseudo-contractions in Banach spaces," Nonlinear Analysis, Theory, Methods and Applications, vol. 70, no. 11, pp. 4039-4046, 2009.

[20] K. Geobel and W. A. Kirk, Topics in Metric Fixed Point Theory, vol. 28, Cambridge Studies in Advanced Mathematics, Cambridge University Press, 1990.

[21] X. Lu, H.-K. Xu, and X. Yin, "Hybrid methods for a class of monotone variational inequalities," Nonlinear Analysis, Theory, Methods and Applications, vol. 71, no. 3-4, pp. 1032-1041, 2009.

[22] H. K. Xu, "Iterative algorithms for nonlinear operators," Journal of the London Mathematical Society, vol. 2, pp. 1-17, 2002. 


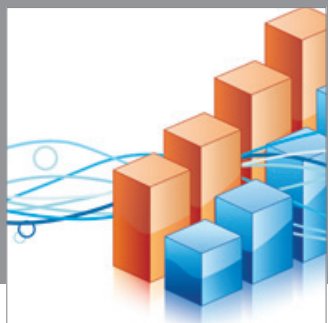

Advances in

Operations Research

mansans

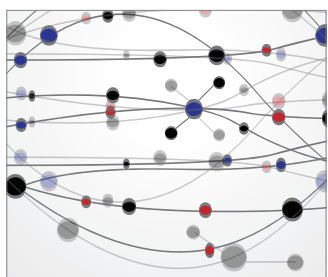

The Scientific World Journal
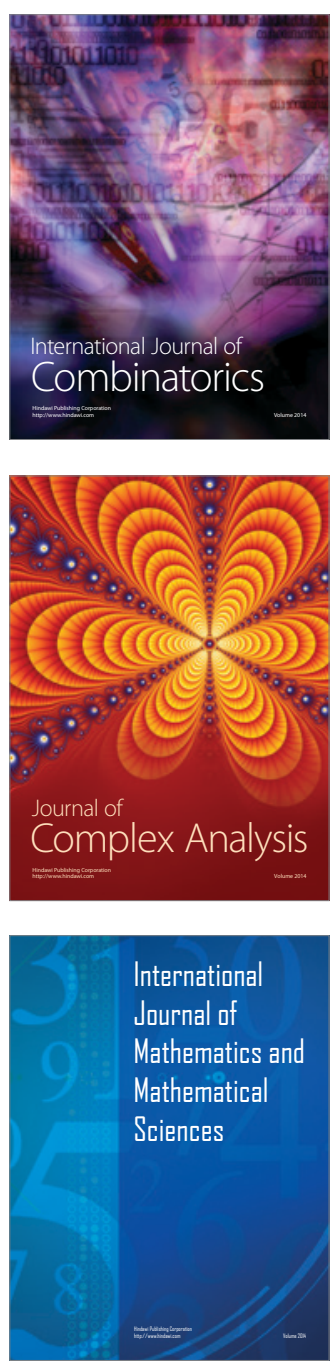
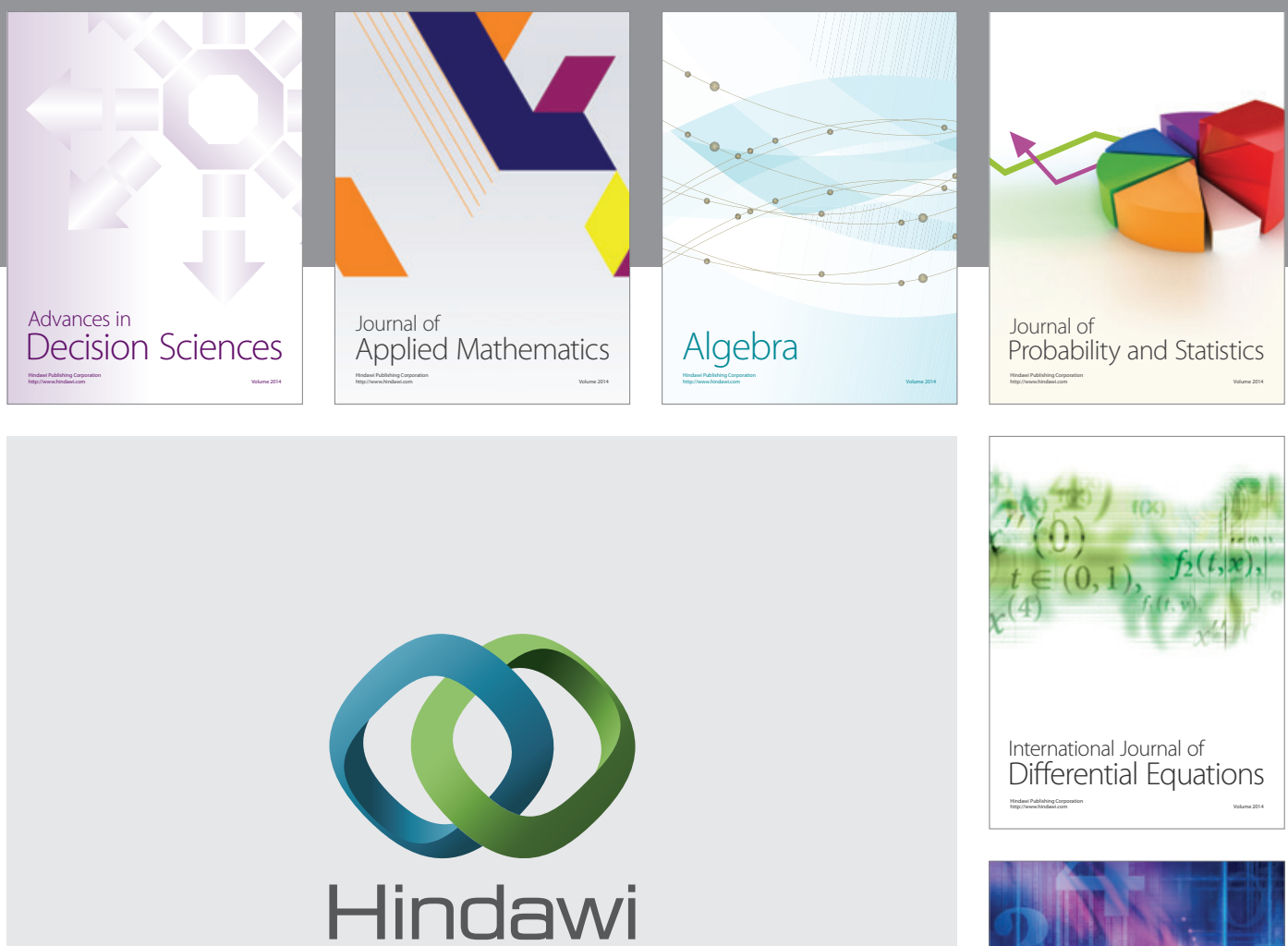

Submit your manuscripts at http://www.hindawi.com
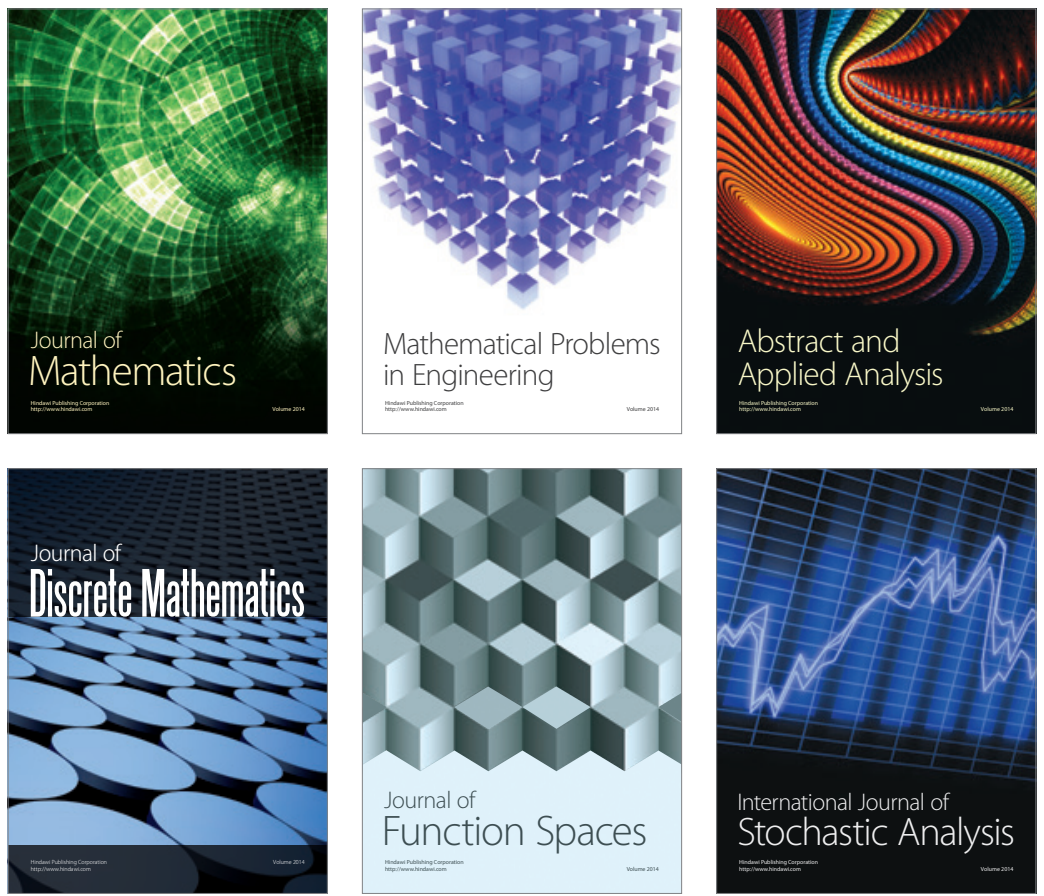

Journal of

Function Spaces

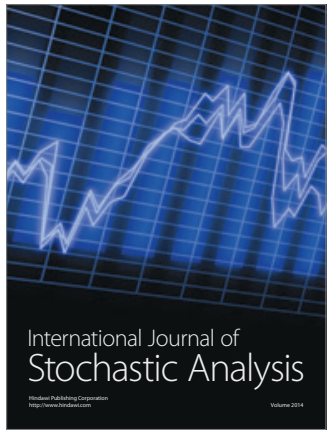

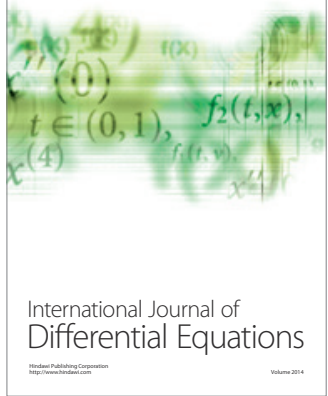
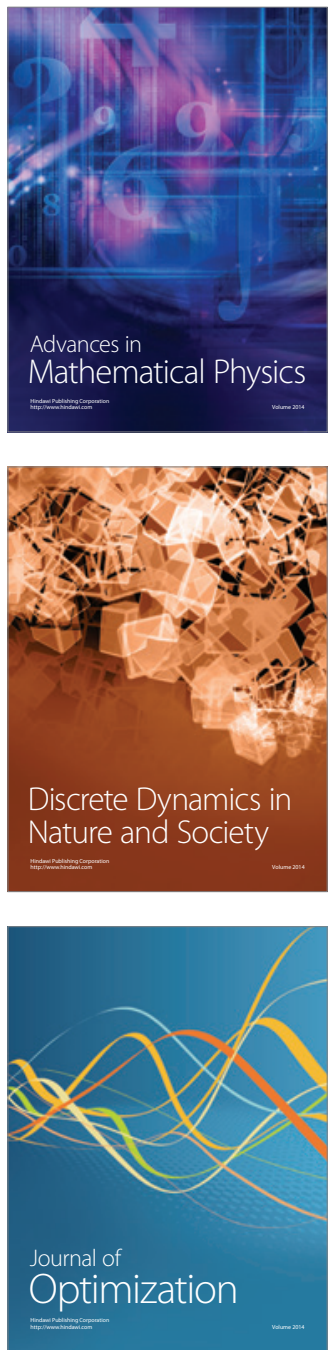